\title{
PERTINENCIA DEL TAMIZAJE GENÉTICO EN DONANTES DE GAMETOS
}

\section{The pertinence of genetic screening in gamete donors}

\author{
Claudia J. Serrano-Serrano, $M D, M S c^{I}$
}

Recibido: marzo 21/12 - Aceptado: septiembre 24/12

\section{RESUMEN}

Introducción: una preocupación importante para las parejas sometidas a tratamientos de reproducción asistida con gametos donados es la posibilidad de adquirir enfermedades infecciosas o la transmisión de enfermedades genéticas a la descendencia. Existen reportes, basados en la legislación de cada país y en la experiencia de cada centro, que hacen referencia al tipo de evaluación genética, infecciosa y psicológica que deben tener los donantes de gametos. En Colombia no existe una ley específica sobre las técnicas de reproducción asistida y la donación de gametos. Tampoco existe claridad acerca de si los donantes deberían ser evaluados genéticamente y con qué tipo de pruebas.

Objetivo: el objetivo de este documento es hacer una reflexión sobre la pertinencia de evaluar genéticamente a los donantes de gametos a partir de la caracterización de las principales condiciones genéticas prevalentes en nuestra población, la alteración genética de base y el método actual de diagnóstico genético.

Conclusión: se concluye que es necesario desarrollar un protocolo claro a nivel nacional y en los centros de reproducción asistida a cerca de la evaluación genética en los pacientes donantes de gametos.

Médico cirujano, Universidad del Rosario. Ginecoobstetra, Universidad del Bosque. Magíster en Genética Humana, Universidad del Rosario. Directora científica del Centro de Investigación en Genética Humana y Reproductiva Genetix - SAS. Bogotá, Colombia. genetix.serrano@gmail.com
Palabras clave: tamizaje genético, donantes de gametos, infertilidad, azoospermia, fibrosis quística, atrofia muscular espinal, síndrome X frágil.

\section{ABSTRACT}

Introduction: A major concern for pairs undergoing assisted reproduction treatment with donated gametes is the possibility of acquiring infectious diseases or transmitting genetic diseases to their offspring. Some reports have been based on the legislation of each country and each centre's experience referring to the type of genetic infectious and/or psychological evaluation which gamete donors must undergo. There is no specific law in Colombia about assisted reproduction and/ or gamete donor techniques; also, there is no clarity about whether donors should be genetically evaluated and which type of test should be used for this.

Objective: This document was aimed at making a reflection about the pertinence of genetically evaluating gamete donors by characterising the main genetic conditions prevailing in the Colombian population, base genetic alteration and the method currently being used for genetic diagnosis.

Conclusion: It was concluded that a clear national protocol must be developed about genetic evaluation in gamete-donating patients; this should also be done in assisted reproduction centres. 
Key words: Genetic screening, gamete donor, infertility, azoospermia, cystic fibrosis, spinal muscular atrophy, fragile $\mathrm{X}$ syndrome.

\section{INTRODUCCIÓN}

Las parejas que se someten a tratamientos de reproducción asistida con gametos donados tienen dos grandes preocupaciones: la posibilidad de adquirir enfermedades infecciosas y la transmisión de enfermedades genéticas a la descendencia. En la actualidad, las principales indicaciones de reproducción asistida con donantes de esperma son: hombres con azoospermia secretora o disfunción eyaculatoria, enfermedades genéticas transmisibles, hombres VIH seropositivos con pareja serodiscordante que deseen evitar cualquier transmisión viral (aunque en algunas instituciones es posible realizar lavados de esperma y fecundación in vitro), pacientes que no puedan permitirse costosos tratamientos de fecundación in vitro pero que requieren de tecnología de reproducción asistida debido a un factor masculino grave, mujeres $\mathrm{Rh}$ negativas isoinmunizadas con parejas Rh positivas y, finalmente, mujeres solteras que desean embarazarse. Para donante de oocitos son: mujeres ooforectomizadas, con falla ovárica precoz o menopaúsicas y portadoras de enfermedades hereditarias (1).

Existen diferentes reportes basados en la legislación de cada país y en la experiencia de cada centro, que hacen referencia al tipo de evaluación, tanto genética como infecciosa y psicológica, que deben tener los donantes (2, 3). En Colombia no existe una ley específica sobre las técnicas de reproducción asistida y la donación de gametos. Estas están contempladas en el Decreto 1546 de 1998, sin que se haga mención de sus consecuencias sobre la filiación, ni las condiciones requeridas para ser receptor(a) de una donación de gametos. Tampoco existe claridad acerca de si los donantes deberían ser evaluados genéticamente y con qué tipo de pruebas (4).

Cada vez con más frecuencia, en la literatura aparecen reportes de condiciones genéticas hereda- das de forma involuntaria de donantes de gametos a parejas receptoras en centros de reproducción asistida (5). Varios autores y en diferentes escenarios han planteado preguntas críticas sobre la viabilidad de las pruebas genéticas en donantes, sus costos e implicaciones éticas (6-8). El uso actual del diagnóstico genético en donantes de gametos es aún inconsistente porque no existe una reglamentación clara para la detección de estas anomalías. La Food and Drug Administration (FDA) en las guías sobre la donación de gametos se enfoca principalmente en agentes infecciosos, con poca atención a los desórdenes genéticos (9). La American Society for Reproductive Medicine (ASRM), en sus guías a cerca de la donación de gametos y embriones recomienda la realización de un cariotipo en sangre a los donantes y screening para fibrosis quística para la mutación Delta F 508. Además, sugiere que se deben realizar pruebas adicionales de acuerdo con los antecedentes personales y familiares, la historia clínica y el grupo poblacional al que pertenezca (10).

El objetivo de este documento es hacer una reflexión sobre la pertinencia de evaluar genéticamente a los donantes de gametos a partir de la caracterización de las principales condiciones genéticas prevalentes en nuestra población, la alteración genética de base y el método actual de diagnóstico genético.

\section{ENFERMEDADES GENÉTICAS FRECUENTES EN COLOMBIA Y A NIVEL INTERNACIONAL}

En general, la enfermedad genética en Colombia tiene una frecuencia que oscila entre el 30 y el 52,8 por cada 1000 habitantes $(11,12)$, dato similar a las cifras reportadas en la literatura mundial. Las malformaciones congénitas de origen multifactorial corresponden al 50\% del total, seguidas por las cromosomopatías y las de origen monogénico. Sin embargo, esta última puede estar sesgada por la falta de diagnóstico genético molecular en el país, y la falta de conocimiento de los clínicos a este respecto (13). En la tabla 1 se describen las enfer- 


\begin{tabular}{|c|c|c|}
\hline $\begin{array}{l}\text { Condiciones autosómicas } \\
\text { dominantes }\end{array}$ & $\begin{array}{c}\text { Condiciones autosómicas } \\
\text { recesivas }\end{array}$ & $\begin{array}{c}\text { Condiciones ligadas al } \\
\text { cromosoma X }\end{array}$ \\
\hline $\begin{array}{l}\text { Riesgo de recurrencia en cada } \\
\text { embarazo es del } 50 \% \text { indepen- } \\
\text { dientemente del sexo del } \\
\text { recién nacido }\end{array}$ & $\begin{array}{l}\text { Riesgo de recurrencia en cada } \\
\text { embarazo es del } 50 \% \text { indepen- } \\
\text { dientemente del sexo del } \\
\text { recién nacido }\end{array}$ & $\begin{array}{l}\text { Riesgo de recurrencia en cada } \\
\text { embarazo es del } 50 \% \text { de hijos } \\
\text { afectados (varones) y } 50 \% \text { de } \\
\text { hijas portadoras }\end{array}$ \\
\hline Hipercolesterolemia familiar & Fibrosis quística & Distrofia muscular de Duchenne \\
\hline Sordera de adulto & Atrofia muscular espinal & Hemofilia A \\
\hline Riñones poliquísticos & Hiperplasia adrenal & $\begin{array}{l}\text { Retardo mental } \\
\text { (Síndrome X Frágil) }\end{array}$ \\
\hline Enfermedad de Huntington & Fenilcetonuria & Ictiosis \\
\hline
\end{tabular}

medades genéticas más diagnosticadas en nuestro país. En la base de datos de Online Inheritance in Man (OMIM) del National Center for Biotechnology Information (NCBI) existen a la fecha 3.414 condiciones de origen monogénico (mendeliano) con fenotipo y genotipo conocido, y 3703 con fenotipo conocido y genotipo que se sospecha mendeliano (http://omim.org/statistics/entry).

\section{Cromosomopatías}

Las alteraciones cromosómicas son variaciones en el número o en la estructura de los cromosomas. Generalmente, estas condiciones se deben a errores durante la gametogénesis o en las primeras divisiones del embrión, y pueden ser heredadas por los padres o ser producto del azar (14). Se estima que cerca de un 60\% de los abortos ocurridos en el primer trimestre de gestación se deben a anomalías cromosómicas, una de cada 250 a 500 personas es portadora de una alteración estructural y un $0,5 \%$ de los recién nacidos presentan aneuploidías (15). Las alteraciones estructurales son causantes además de aborto a repetición, retardo mental y malformaciones congénitas (16). Algunas de las alteraciones halladas en donantes asintomáticos son cromosomas marcadores, trisomías en mosaico o translocaciones balanceadas y recíprocas. Este tipo de alteraciones se estudian mediante el análisis del cariotipo; esta prueba es la que con mayor frecuencia se realiza en la actualidad en los centros de reproducción asistida para el estudio genético de los donantes de gametos (10).

\section{Enfermedades autosómicas recesivas}

En las enfermedades autosómicas recesivas (AR) un individuo debe tener mutaciones en ambas copias de su gen para manifestar la enfermedad. Estos alelos mutados provienen de sus padres, que habitualmente son heterocigotos sanos (poseen una copia normal del gen y una mutada). Si ambos miembros de una pareja son portadores sanos, tienen un 25\% de probabilidad de tener un hijo afectado y esta cifra permanece igual para cada embarazo. Con mayor frecuencia se asocian a consanguinidad, grupos étnicos con alta endogamia o regiones geográficas con poca migración poblacional (17). A continuación se describen las más prevalentes en Colombia.

Fibrosis quística $(F Q)$ : es la enfermedad AR más común en la población caucásica. Cursa con compromiso multisistémico, crónico y progresivo. $\mathrm{Su}$ principal sintomatología es pulmonar, con infecciones a repetición pero también puede tener afecta- 
ción pancreática y gastrointestinal, y azoospermia obstructiva por agenesia de los conductos deferentes en el varón (18). La incidencia oscila entre 1:1900 a 1:3700 recién nacidos vivos, con una frecuencia de portadores del 5\% en población caucásica. En asiáticos y afroamericanos la incidencia es de 1:32.000 y 1:15.000 recién nacidos vivos respectivamente (19). En Colombia se estima que la tasa de portadores es de 1 de cada 47 personas (19). El gen CFTR está ubicado en el cromosoma 7 y se han descrito más de 1700 mutaciones causantes de la enfermedad, con frecuencias individuales variables de acuerdo con la población estudiada (18). La mutación más común es la deleción de fenilalanina en la posición 508 de la proteína y se observa en el $85 \%$ de los caucásicos y en el 28-42\% de la población colombiana $(18,20)$. Existen adicionalmente 25 mutaciones frecuentes en caucásicos y son las que el Colegio Americano de Genética recomienda realizar en el estudio de donantes de gametos en algunas instituciones en Estados Unidos (21). En Colombia las más frecuentes son: $1811+1,6 \mathrm{kbA}>\mathrm{G}, 621+1 \mathrm{G}>\mathrm{T}$ y G542 X y representan el 9\% de la población (22). Mediante pruebas de biología molecular -reacción en cadena de la polimerasa (PCR) - es posible detectar la mutación Delta F508, y en los casos en que esta es negativa y si la historia clínica lo amerita, se continúa con el estudio de las 25 mutaciones recomendadas por el Colegio Americano de Genética o con la secuenciación del gen completo $(22,23)$.

Atrofia Muscular Espinal (AME): son un grupo heterogéneo de enfermedades heredadas que se caracterizan por la degeneración de las neuronas motoras de las astas anteriores de la médula espinal con atrofia muscular, hipotonía, arreflexia y parálisis progresiva (24). Después de la fibrosis quística es el trastorno AR más común, con una tasa de portadores de 1 en 35-60 personas según diferentes poblaciones. Afecta a uno de cada 6000 a 10.000 niños, y es la causa genética más frecuente de mortalidad infantil (25). Existen tres subtipos clínicos de AME AR de acuerdo con la edad de presentación, la forma más severa (tipo I) es la
Enfermedad de Werdnig-Hofmann, con afectación incluso desde la vida intrauterina (26). En el 95\% de los casos es causada por una deleción en los exones 7 y 8, con mayor frecuencia del 7, del gen SMN1 (Survival Motor Neuron 1). En el 5\% restante se debe a mutaciones puntuales de este gen. El gen SMN1 se localiza en la región telomérica del cromosoma 5q11.2 y posee un pseudogen, el SMN2, de localización centromérica. A mayor número de copias de SMN2, menos severa es la enfermedad $(26,27)$. En la actualidad se cuenta con diferentes técnicas moleculares para realizar el diagnóstico de afectados y de portadores analizando el número de copias de los genes SMN1 y SMN2 (28).

Hemoglobinopatías: son defectos en la estructura de una de las cadenas de globina de la molécula de hemoglobina. La condición más frecuente es la anemia de células falciformes, y se estima que el 7\% de la población africana es portadora. También es frecuente en el sureste asiático y el mediterráneo. Se caracteriza por la presencia de hemoglobina $S$ (HbS) (29). La hemoglobina humana normal es un tetrámero compuesto de dos cadenas de hemoglobina $\alpha$ y dos $\beta$. La HbS es el resultado de una mutación puntual en el gen HBB (Glu6Val). Esta anemia puede resultar de la combinación de $\mathrm{HbS}$ con otras variantes de cadena $\beta$-globina anormal o con talasemias (30). Las talasemias son defectos que se caracterizan por una disminución en la cantidad de cadenas de globina (31). El diagnóstico de afectados y de portadores de estas condiciones se realiza mediante electroforesis de hemoglobina o cromatografía líquida (HPLC) (32). Una vez se realiza el diagnóstico es posible analizar mutaciones desde el punto de vista molecular para ofrecer diagnóstico prenatal o preimplantacional.

\section{Enfermedades ligadas al cromosoma $\mathbf{X}$}

Las enfermedades monogénicas ligadas al cromosoma $\mathrm{X}$, en general afectan únicamente a los hombres ya que solo cuentan con un cromosoma $\mathrm{X}$. Las mujeres son portadoras pero no afectadas, y transmitirán la enfermedad al 50\% de sus hijos, 
quienes expresarán el fenotipo, y la mitad de sus hijas serán portadoras. Los hombres transmiten la enfermedad al 100\% de sus hijas, quienes serán portadoras (33).

Síndrome $X$ frágil: el síndrome de $\mathrm{X}$ frágil no es solo la causa más común de retraso mental heredado en varones sino que algunas formas alélicas pueden causar baja respuesta ovárica y falla ovárica prematura en mujeres (FOP) (34). Este trastorno está asociado con una expansión dinámica de tripletas CGG en el gen FMR1 localizado en el cromosoma X. La mutación completa consiste en alelos con más de 200 repeticiones CGG, que actúa negativamente en la transcripción del gen silenciándolo (35). Las mujeres portadoras de la premutación presentan una expansión que oscila entre 55 y 199 repeticiones, y se ha visto asociada a insuficiencia ovárica (36). Las expansiones en rangos intermedios son alelos con 45 a 54 repeticiones que, aunque son clínicamente asintomáticos, pueden también expandirse de forma inestable a las siguientes generaciones. En la población general el pico de repeticiones se encuentra entre 29 y $30(37,38)$. Se estima que una de cada 130-500 mujeres (1,3\%) son portadoras de la premutación según diferentes estudios (39, 40). La PCR se utiliza para la medición del número de repeticiones CGG en el gen FMR1. Esta técnica permite evaluar hasta 110 repeticiones. En los casos en que solo se observa un alelo en mujeres, se debe descartar que sean homocigotas o portadoras de la premutación o mutación mediante estudios de cigocidad analizando los padres o mediante SouthernBlot. Este último permite medir todas las repeticiones independientemente de su número. La técnica de PCR está aceptada como prueba de screening en la actualidad (41).

Distrofia muscular de Duchenne: las distrofias musculares (DM) son entidades hereditarias que se caracterizan por debilidad muscular progresiva, pérdida de la masa muscular, hiporreflexia, fasciculaciones y discapacidad física variable. La distrofia muscular de Duchenne (DMD) es la más común, y afecta a uno de cada 3500 niños del sexo masculino. La distrofia muscular de Becker (DMB) es menos frecuente y se caracteriza por una progresión más lenta y mayor sobrevida (42). El gen de la distrofina se localiza en el brazo corto del cromosoma X. La literatura mundial muestra que 66\% de todas las mutaciones corresponden a deleciones o duplicaciones de uno o más exones en el gen. Las deleciones tienen una distribución no al azar y se agrupan en dos regiones proclives. El 80\% se concentran en la región de los exones 44 al 52, y el 20\% restante comprenden los exones 1 al 19 (43). Debido al agrupamiento de las deleciones en dos puntos proclives se ha establecido que el análisis de 18 de los 79 exones permite identificar el 98\% de todas las deleciones. En el 33\% de los casos se trata de mutaciones puntuales o de pocos nucleótidos (44). El diagnóstico de portadoras es posible mediante análisis de las mutaciones cuando existe un caso en la familia, o mediante un análisis indirecto con personas sanas y enfermas de una misma familia para evaluar en cuál cromosoma $\mathrm{X}$ se encuentra la mutación (45).

\section{CONCLUSIONES}

De acuerdo con la prevalencia de enfermedades genéticas en Colombia las pruebas que se deberían realizar de forma rutinaria a pacientes donantes de gametos varían según los grupos poblacionales. La diversidad de la población colombiana, con sus variados grupos étnicos, hace difícil elegir las pruebas que se deben practicar. Probablemente sea beneficiosa la valoración por un genetista, la realización de un cariotipo, un análisis molecular de fibrosis quística, atrofia muscular espinal, síndrome $\mathrm{X}$ frágil y una electroforesis de hemoglobina. Otras pruebas podrían ser pertinentes de acuerdo con los antecedentes de cada paciente. Por ejemplo, en el caso de personas de origen asiático o mediterráneo también se deben descartar talasemias. En aquellas de origen africano, anemia de células falciformes; y en judíos, enfermedad de TaySachs, enfermedad de Gaucher, enfermedad de Canavan, deficiencia de Alfa 1 Antitripsina, anemia de Fanconi, fibrosis quística y síndrome de Bloom, entre otras. 
Un cuidadoso asesoramiento genético a los pacientes donantes de gametos es recomendable por parte de un genetista ya que pueden existir antecedentes familiares y personales así como características demográficas tales como raza, sexo o lugar de origen que pueden sugerir la existencia de una enfermedad genética transmisible. Asimismo, se deben conocer las limitaciones de las pruebas genéticas y brindar tranquilidad acerca del bajo riesgo que representan las técnicas de reproducción asistida.

No está claro qué norma sería efectiva para el estudio de estos donantes ya que la mayoría de trastornos genéticos pueden surgir de novo, así como de eventos multifactoriales asociados al ambiente. Además, también hay que tener en cuenta el impacto en los costos para las parejas receptoras. Hay que recordar que la realización de pruebas genéticas a pacientes incluidos en programas de donación de gametos pueden reducir el riesgo de enfermedades específicas pero no pueden eliminar del todo el 3-4\% de riesgo para defectos congénitos que tienen todos los recién nacidos.

Por tanto, es necesario desarrollar un protocolo claro a nivel nacional y en los centros de reproducción asistida a cerca de la evaluación genética en los pacientes donantes de gametos. Se debe brindar un cuidadoso asesoramiento genético a los pacientes donantes de gametos y parejas receptoras por parte de un genetista.

\section{REFERENCIAS}

1. Garrido N, Zuzuarregui JL, Meseguer M, Simón C, Remohí J, Pellicer A. Sperm and oocyte donor selection and management: experience of a 10 year follow-up of more than 2100 candidates. Hum Reprod 2002;17:3142-8.

2. Daar J, Brzyski R. Genetic screening of sperm and oocyte donorsethical and policy implications. JAMA 2009;302:1702-4.

3. Sims CA, Callum P, Ray M, Iger J, Falk RE. Genetic testing of sperm donors: survey of current practices. Fertil Steril 2010;1:126-9.

4. Decreto 1546 de 1998 (agosto 4). Diario Oficial
43.357, del 6 de agosto de 1998, Ministerio de Salud Píblica, Leyes 9 de 1979 y 73 de 1988, en cuanto a la obtención, donación, preservación, almacenamiento, transporte, destino y disposición final de componentes anatómicos y los procedimientos para trasplante de los mismos en seres humanos, y se adoptan las condiciones mínimas para el funcionamiento de las Unidades de Biomedicina Reproductiva, Centros o similares.

5. Molina O, Blanco J, Vidal F. Deletions and duplications of the 15q11-q13 region in spermatozoa from Prader-Willi syndrome fathers. Mol Hum Reprod 2010;16:320-8.

6. Maron BJ, Lesser JR, Schiller NB, Harris KM, Brown C, Rehm HL. Implications of hypertrophic cardiomyopathy transmitted by sperm donation. JAMA 2009;302:1681-4.

7. Callum P, Iger J, Ray M, Sims CA, Falk RE. Outcome and experience of implementing spinal muscular atrophy carrier screening on sperm donors. Fertil Steril 2010;94:1912-4.

8. Callum P, Urbina MT, Falk RE, Alvarez-Diaz JA, Benjamin I, Sims CA. Spinal muscular atrophy (SMA) after conception using gametes from anonymous donors: recommendations for the future. Fertil Steril 2010;93:1006.e1-2.

9. U.S. Food and Drug Administration (FDA). FDA Regulation of Human Cells, Tissues, and Cellular and Tissue-Based Products. Disponible en: http//www.fda. gov/BiologicsBloodVaccines/TissueTissueProducts/ RegulationofTissues/ucm150485.htm

10. Practice Committee of American Society for Reproductive Medicine; Practice Committee of Society for Assisted Reproductive Technology. 2008 Guidelines for gamete and embryo donation: a Practice Committee report. Fertil Steril 2008;5 Suppl:S30-44.

11. Páez P, Suárez-Obando F, Zarante I. Enfermedades de origen genético en pacientes pediátricos hospitalizados en la Provincia de Ubaté, Colombia. Salud Pública 2008;10:414-22.

12. Bernal J, Suárez F. La carga de la enfermedad genética en Colombia, 1996-2025. Univ Med Bogotá (Colombia) 2005;49:12-28. 
13. Zarante I, Franco L, López C, Fernández N. Frecuencia de malformaciones congénitas. Evaluación y pronóstico de 52.744 nacimientos en cuatro ciudades colombianas. Biomédica 2010;30:65-71.

14. Schaeffer A, ChungJ, Heretis K, Wong A, Ledbetter DH, Lese Martin C. Comparative genomic hybridizationarray analysis enhances the detection of aneuploidies and submicroscopic imbalances in spontaneous miscarriages. Am J Hum Genet 2004;74:1168-74.

15. Chen CP, Wu PC, Lin CJ, Chern SR, Tsai FJ, Lee C, et al. Unbalanced reciprocal translocation at amniocentesis. Taiwanese J Obstet Gynecol 2011;50:48-57.

16. Vandeweyer G, Kooy RF. Balanced translocations in mental retardation. Hum Genet 2009;126:133-47.

17. Health Centre for Genetics Education. Autosomal Recessive Inheritance. Disponible en: http://www. genetics.edu.au/Information/Genetics-Fact-Sheets/ Autosomal-Recessive-Inheritance-FS8/view

18. Cystic Fibrosis Mutation Database. Disponible en: http://www.genet.sickkids.on.ca/cftr/StatisticsPage.html

19. Lay-Son G, Repetto G. Genética y fibrosis quística: desde el gen CFTR a los factores modificadores. Neumología pediátrica 2010;5:4-9.

20. Malambo D, Gómez D, Veloza L, Arbeláez J, Gómez C. Algunos aspectos hereditarios y ambientales en casos de fibrosis quística en la ciudad de Cartagena (Colombia). Salud Uninorte. Barranquilla (Col) 2008;24:10-22.

21. Ortigosa L. Fibrosis quística: aspectos diagnósticos. Colombia Médica 2007;38:Supl 1.

22. Dequeker E, Stuhrmann M, Morris MA, Casals T, Castellani C, Claustres M, et al. Best practice guidelines for molecular genetic diagnosis of cystic fibrosis and CFTR-related disorders--updated European recommendations. Eur J Hum Genet 2009; 17:51-65.

23. Vásquez C, Aristizábal R, Daza W. Fibrosis quística en Colombia. Neumología pediátrica 2010;5:44-50.

24. Keyeux G, Rodas C, Bienvenu T, Garavito P, Vidaud D, Sanchez D, et al. CFTR mutations in patients from Colombia: implicactions for local and regional molecular diagnosis programs. Hum Mut 2003;22:1-7.
25. Ogino S, Wilson R. Spinal muscular atrophy: molecular genetics and diagnostics. Expert Rev Mol Diagn 2004;4:15-29.

26. Monani U. Spinal muscular atrophy: a deficiency in a ubiquitous protein; a motor neuron-specific disease. Neuron 2004;48:885-96.

27. Akutsu T, Nishio H, Sumino K, Takeshima Y, Tsuneishi S, Wada H, et al. Molecular genetics of spinal muscular atrophy: contribution of the naip gene to clinical severity. Kobe J Med Sci 2002;48:25-31.

28. McAndrew PE, Parsons W, Simard LR, Rochette C, Ray PN, Mendell JR, et al. Identification of proximal spinal muscular atrophy carriers and patients by analysis of SMNT and SMNC gene copy number. Am J Hum Genet 1997;60:1411-22.

29. Stewart H, Wallace A, McGaughran J, Mountford R, Kingston H. Molecular diagnosis of spinal muscular atrophy. Arch Dis Child 1998;78:531-5.

30. Steinberg MH, Adewoye AH. Modifier genes and sickle cell anemia. Curr Opin Hematol 2006;13:131-6.

31. Clark BE, Thein SL. Molecular Diagnosis of Haemoglobin Disorders. Clin Lab Haematol 2004; 26:159-76.

32. Troxler H, Kleinert P, Schmugge M, Speer O. Advances in hemoglobinopathy detection and identification. Adv Clin Chem 2012;57:1-28.

33. Health Centre for Genetics Education. X-Linked Inheritance. Disponible en: http://www.genetics. edu.au/Information/Genetics-Fact-Sheets/X-LinkedInheritance

34. Rousseau F, Rouillard P, Morel ML, Khandjian EW, Morgan K. Prevalence of carriers of premutation-size alleles of the FMR1 gene--and implications for the population genetics of the fragile $\mathrm{X}$ syndrome. Am J Hum Genet 1995;57:1006-18.

35. Bennett CE, Conway GS, Macpherson JN, Jacobs PA, Murray A. Intermediate sized CGG repeats are not a common cause of idiopathic premature ovarian failure. Hum Reprod 2010;25:1335-8.

36. Lyon E, Laver T, Yu P, Jama M, Young K, Zoccoli M, et al. A simple, high-throughput assay for Fragile X expanded alleles using triple repeat primed PCR and capillary electrophoresis. J Mol Diagn 2010;12:505-11. 
37. Kremer EJ, Pritchard M, Lynch M, Yu S, Holman K, Baker E, et al. Mapping of DNA instability at the fragile $\mathrm{X}$ to a trinucleotide repeat sequence $\mathrm{p}(\mathrm{CCG}) \mathrm{n}$. Science 1991;252:1711-4.

38. Chatterjee S, Maitra A, Kadam S, Patel Z, Gokral J, Meherji P. CGG repeat sizing in the FMR1 gene in Indian women with premature ovarian failure. Reprod Biomed Online 2009;19:281-6.

39. Gleicher N, Weghofer A, Oktay K, Barad D. Relevance of triple CGG repeats in the FMR1 gene to ovarian reserve. Reprod Biomed Online 2009; 19:385-90.

40. Lledo B, Guerrero J, Ortiz JA, Morales R, Ten J, Llacer $\mathrm{J}$, et al. Intermediate and normal sized CGG repeat on the FMR1 gene does not negatively affect donor ovarian response. Hum Reprod 2012; 27:609-14.

41. Strom CM, Crossley B, Redman JB, Buller A, Quan F, Peng M, et al. Molecular testing for Fragile X Syndrome: lessons learned from 119,232 tests performed in a clinical laboratory. Genet Med 2007;9:46-51.

42. Mercuri E, Muntoni F. The ever-expanding spectrum of congenital muscular dystrophies. Ann Neurol 2012;72:9-17.

43. Silva C, Fonseca D, Restrepo CM, Contreras NC, Mateus HE. Deleciones en el gen de la distrofina en 62 familias colombianas: correlación genotipo- fenotipo para la distrofia muscular de Duchenne y Becker. Colomb Med 2004;35:191-8.

44. Lovering RM, Porter NC, Bloch RJ. The muscular dystrophies: from genes to therapies. Physther 2005;85:1372-88.

45. Bakker E, Hofker MH, Goor N, Mandel JL, Wrogemann K, Davies KE, et al. Prenatal diagnosis and carrier detection of Duchenne muscular dystrophy with closely linked RFLP's. Lancet 1985;1:655-8. 